\title{
Flow past an Electrically Conducting Fluid over a Vertical Oscillating Plate with Chemical Reaction.
}

\author{
Bhaben Ch. Neog ${ }^{1}$, Rudra Kr. Das ${ }^{2}$ \\ ${ }^{1}$ Applied Mathematics Research Centre, Jagiroad College, Jagiroad, Assam. \\ ${ }^{2}$ Dept. of Physics, Jagiroad College, Jagiroad, Morigaon, Assam.
}

\begin{abstract}
Effect of magnetic field on transient free convection flow past an electrically conducting fluid over an oscillating vertical plate with chemical reaction is studied here. Exact solutions obtained by Laplace Transform methods are presented graphically for different values of physical parameters. It is observed that chemical reaction parameter and magnetic parameter influence the velocity and concentration profiles significantly.

Keywords: Free Convection, MHD, Oscillating Plate, Chemical Reaction AMS 2000 subject classification: 76R10, 76W05, 80A20, 80A32
\end{abstract}

\section{Introduction}

Because of many industrial and technological applications many authors found interest to investigate heat and mass transfer problems. Mass transfer with chemical reaction is another most commonly encountered circumstance in chemical industry as well as in physical and biological sciences. In some other areas such as food processing industry, paper processing technology, the evaporation or condensation process, solvent extraction, drying humidification, sublimation, oxygenation of blood, food and drug assimilation, respiration mechanism, etc. chemical reaction takes place. There are many situations where convection heat transfer phenomena are accompanied by mass transfer also. When mass transfer takes place in a fluid at rest, the mass is transferred purely by molecular diffusion resulting from concentration gradients. For low concentration of the mass in the fluid and low mass transfer rates, the convective heat and mass transfer processes are similar in nature. A number of investigations have already been carried out with combined heat and mass transfer under the assumption of different physical situations.

Further due to some important industrial and engineering applications such as liquid metal cooling in nuclear reactors, magnetic control of molten iron flow in steel industry etc., magneto-convection has also been gaining considerable attention amongst researchers. Hence combined study will surely enhance the already developed areas further for more complex studies.

Exact solutions of free convection flow past a vertical oscillating plate in free convective flow was first obtained by Soundalgekar [14] and the same problem with mass transfer effect was considered by Soundalgekar and Akolkar [15]. Das et. al. [3] studied the effects of mass transfer on free convection flow past an impulsively started infinite vertical plate with constant heat flux and chemical reaction. They also studied the transient free convection flow past an infinite vertical plate with periodic temperature [5]. Effect of mass transfer on the flow past an infinite vertical oscillating plate with constant heat flux was studied by Soundalgekar et. al. [17].

The effects of mass transfer on free convection flow past a semi-infinite vertical isothermal plate was first studied by Gebhart and Pera [10] and the effects of mass transfer on the flow past an impulsively started infinite vertical plate with variable temperature was studied by Soundalgekar et. al. [16]. Muthucumaraswamy et. al. [12] considered the effects of mass transfer on impulsively started infinite vertical plate with variable temperature and uniform mass flux. All of them considered the fact that free convection current caused by temperature differences is also caused by the differences in concentration or material constitution as suggested by Gebhart [9].

Further, in many cases in the process of free convection, chemical reaction also takes place due to the presence of foreign masses as impurities in fluid. It is found that in many chemical engineering processes, chemical reaction takes place between foreign masses, present in the form of ingredients and the fluid. This type of chemical reaction may change the temperature and the heat content of the fluid and may affect the free convection process. However, if the presence of such foreign mass is very low then we can assume the first order chemical reaction so that heat generation due to chemical reaction can be considered to be very negligible. Das et. al. [4] considered the effects of mass transfer on flow past an impulsively started vertical plate and Muthucumaraswamy and Meenakshisundaram [13] studied the chemical reaction effects on vertical oscillating plate with variable temperature and chemical reaction. Deka and Neog [6] considered the combined effects of thermal radiation and chemical reaction on free convection flow past a vertical plate in porous medium. Chaudhary and Jain [2] studied the magneto-hydrodynamic transient heat and mass transfer flow by free convection past a vertical plate, when the temperature of the plate oscillates in time about a constant mean 
temperature and the plate is embedded in a porous medium. They extended the work of Das et.al. [3], which include the effects of mass transfer, magnetic field and porous medium. Recently, Neog [8] studied the unsteady MHD flow past a vertical oscillating plate with variable temperature and chemical reaction and Deka and Neog [7] studied the MHD flow past a vertical oscillating plate with thermal radiation and variable mass diffusion.

Although different authors studied mass transfer with or without chemical reaction in flow past oscillating vertical plate by considering different surface conditions but the study on the effects of magnetic field on free convection heat and mass transfer in the presence of chemical reaction and oscillating plate has not been found in literature and hence the motivation to undertake this study. It is therefore proposed to study the effects of chemical reaction on hydromagnetic flow past an oscillating vertical plate under the assumption of first order chemical reaction.

\section{Mathematical Analysis}

We have considered here an unsteady natural convection flow of a viscous incompressible electrically conducting fluid past an infinite vertical plate. To visualize the flow pattern a Cartesian co-ordinate system is considered where $x^{\prime}$-axis is taken along the infinite vertical plate, $y^{\prime}$-axis is normal to the plate and fluid fills the region $y^{\prime} \geq 0$. Initially, the fluid and the plate are kept at the same constant temperature $T_{\infty}^{\prime}$ and species concentration $C_{\infty}^{\prime}$. At time $t^{\prime}>0$, the plate is given an oscillatory motion in its own plane with a velocity $U_{0} \cos \omega^{\prime} t^{\prime}$. At the same time the plate temperature is raised to $T^{\prime}{ }_{w}$ and concentration is raised to $C^{\prime}{ }_{w}$ and a magnetic field of uniform strength $B_{0}$ is applied normal to the plate. It is assumed that the magnetic Reynolds number is very small and the induced magnetic field is negligible in comparison to the transverse magnetic field. It is also assumed that the effect of viscous dissipation is negligible in the energy equation and the level of species concentration is very low so the Soret and Dufour effects are negligible.

As the plate is infinite in extent so the derivatives of all the flow variables with respect to $x^{\prime}$ vanish and they can be assumed to be functions of $y^{\prime}$ and $t^{\prime}$ only as a result the motion becomes one dimensional with only non-zero vertical velocity component $u^{\prime}$, varying with $y^{\prime}$ and $t^{\prime}$ only. Due to one dimensional nature, the equation of continuity is trivially satisfied.

Under the above assumptions and following Boussinesq approximation, the unsteady flow field is governed by the following set of equations:

$$
\begin{aligned}
& \frac{\partial u^{\prime}}{\partial t^{\prime}}=g \beta\left(T^{\prime}-T_{\infty}^{\prime}\right)+g \beta^{*}\left(C^{\prime}-C_{\infty}^{\prime}\right)+v \frac{\partial^{2} u^{\prime}}{\partial y^{\prime 2}}-\frac{\sigma B_{0}{ }^{2}}{\rho} u^{\prime} \\
& \rho C_{p} \frac{\partial T^{\prime}}{\partial t^{\prime}}=k \frac{\partial^{2} T^{\prime}}{\partial y^{\prime 2}} \\
& \frac{\partial C^{\prime}}{\partial t^{\prime}}=D \frac{\partial^{2} C^{\prime}}{\partial y^{\prime 2}}-K_{l} C^{\prime}
\end{aligned}
$$

Along with the following initial and boundary conditions:

$$
\begin{aligned}
& u^{\prime}=0, \quad T^{\prime}=T_{\infty}^{\prime}, \quad C^{\prime}=C_{\infty}^{\prime} \text { for all } y^{\prime} \text { and } t^{\prime} \leq 0
\end{aligned}
$$

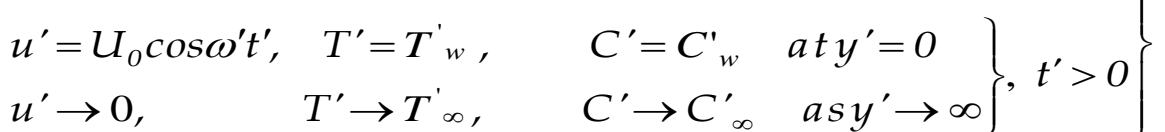

Now to reduce the above equations in non-dimensional form we introduce the following non-dimensional quantities.

$$
\begin{aligned}
& u=\frac{u^{\prime}}{U_{0}}, \quad t=\frac{t^{\prime} U_{o}^{2}}{v}, \quad y=\frac{y^{\prime} U_{0}}{v}, \quad \theta=\frac{T^{\prime}-T_{\infty}^{\prime}}{T^{\prime}{ }_{w}-T_{\infty}^{\prime}}, \quad \phi=\frac{C^{\prime}-C_{\infty}^{\prime}}{C^{\prime}{ }_{w}-C_{\infty}^{\prime}}, \\
& \operatorname{Pr}=\frac{\mu C_{p}}{\kappa}, \quad G r=\frac{g \beta v\left(T_{w}^{\prime}-T_{\infty}^{\prime}\right)}{U_{o}{ }^{3}}, \quad G m=\frac{g \beta^{*} v\left(C_{w}^{\prime}-C_{\infty}^{\prime}\right)}{U_{o}{ }^{3}}, \\
& R=\frac{v K_{1}}{U_{o}^{2}}, \quad S c=\frac{v}{D}, \quad \omega=\frac{\omega^{\prime} v}{U_{o}^{2}}, \quad M=\frac{\sigma B_{0}{ }^{2} v}{\rho U_{o}{ }^{2}}
\end{aligned}
$$

Thus the equations (1), (2) and (3) then reduce to:

$$
\frac{\partial u}{\partial t}=\frac{\partial^{2} u}{\partial y^{2}}+G r \theta+G m \phi-M u
$$




$$
\begin{aligned}
& \frac{\partial \theta}{\partial t}=\frac{1}{\operatorname{Pr}} \frac{\partial^{2} \theta}{\partial y^{2}} \\
& \frac{\partial \phi}{\partial t}=\frac{1}{S c} \frac{\partial^{2} \phi}{\partial y^{2}}-R \phi
\end{aligned}
$$

And the initial and boundary conditions are as follows:

$$
\begin{aligned}
& u=0, \quad \theta=0, \quad \phi=0, \text { for all yand } \leq 0
\end{aligned}
$$

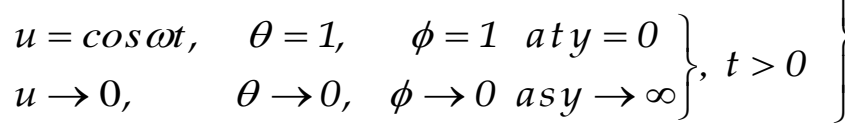

Solutions of the equations (6), (7) and (8) subject to the initial and boundary conditions (9) are obtained with the help of Abramowtz and Stegum [1] and Hetnarski's [9] algorithm. They are obtained as follows:

$$
\begin{aligned}
\theta(y, t)= & \operatorname{erfc}\left(\frac{y \sqrt{\mathrm{Pr}}}{2 \sqrt{t}}\right) \\
\phi(y, t)= & \frac{1}{2}\left\{e^{-y \sqrt{S c R}} \operatorname{erfc}\left(\frac{y \sqrt{S c}}{2 \sqrt{t}}-\sqrt{R t}\right)+e^{y \sqrt{S c R}} \operatorname{erfc}\left(\frac{y \sqrt{S c}}{2 \sqrt{t}}+\sqrt{R t}\right)\right\} \quad(11) \\
u(y, t)= & \frac{1}{4}\left[e^{i \omega t}\left\{e^{-y \sqrt{a}} \operatorname{erfc}\left(\frac{y}{2 \sqrt{t}}-\sqrt{a t}\right)+e^{y \sqrt{a}} \operatorname{erfc}\left(\frac{y}{2 \sqrt{t}}+\sqrt{a t}\right)\right\}+c c\right] \\
& +\frac{G_{1}}{2 b}\left[e ^ { b t } \left(\left\{e^{-y \sqrt{c}} \operatorname{erfc}\left(\frac{y}{2 \sqrt{t}}-\sqrt{c t}\right)+e^{y \sqrt{c}} \operatorname{erfc}\left(\frac{y}{2 \sqrt{t}}+\sqrt{c t}\right)\right\}\right.\right. \\
& \left.\left.-\left\{e^{-y \sqrt{b P r}} \operatorname{erfc}\left(\frac{y \sqrt{P r}}{2 \sqrt{t}}-\sqrt{b t}\right)-e^{y \sqrt{b P r}} \operatorname{erfc}\left(\frac{y \sqrt{P r}}{2 \sqrt{t}}+\sqrt{b t}\right)\right\}\right)\right] \\
& \frac{G_{2}}{2 d}\left[e ^ { d t } \left(\left\{e^{-y \sqrt{f}} \operatorname{erfc}\left(\frac{y}{2 \sqrt{t}}-\sqrt{f t}\right)+e^{y \sqrt{f}} \operatorname{erfc}\left(\frac{y}{2 \sqrt{t}}+\sqrt{f t}\right)\right\}\right.\right. \\
& \left.-\left\{e^{-y \sqrt{h S c}} \operatorname{erfc}\left(\frac{y \sqrt{S c}}{2 \sqrt{t}}-\sqrt{h t}\right)+e^{y \sqrt{h S c}} \operatorname{erfc}\left(\frac{y \sqrt{S c}}{2 \sqrt{t}}+\sqrt{h t}\right)\right\}\right) \\
& \left.-\left\{e^{-y \sqrt{S c R}} \operatorname{erfc}\left(\frac{y \sqrt{S c}}{2 \sqrt{t}}-\sqrt{R t}\right)+e^{y \sqrt{S c R}} \operatorname{erfc}\left(\frac{y \sqrt{S c}}{2 \sqrt{t}}+\sqrt{R t}\right)\right\}\right] \\
& -\frac{G_{3}}{2}\left\{e^{-y \sqrt{M}} \operatorname{erfc}\left(\frac{y}{2 \sqrt{t}}-\sqrt{M t}\right)+e^{y \sqrt{M}} \operatorname{erfc}\left(\frac{y}{2 \sqrt{t}}+\sqrt{M t}\right)\right\} \\
+ & \frac{G_{1}}{b} \operatorname{erfc}\left(\frac{y \sqrt{P r}}{2 \sqrt{t}}\right)
\end{aligned}
$$

Here, the following symbols are used in the above solutions:

$$
\begin{aligned}
& a=M+i \omega, \quad b=\frac{M}{P r-1}, \quad c=M+b, \quad d=\frac{R S c-M}{1-S c}, \quad f=M+d, \\
& h=R+d, \quad G_{1}=\frac{G r}{P r-1}, G_{2}=\frac{G m}{S c-1}, \quad G_{3}=\frac{G_{2}}{d}+\frac{G_{1}}{b}
\end{aligned}
$$

\section{Results and Discussion}

To know the effect of physical parameters viz., chemical reaction parameter, Schmidt number, thermal Grashof number, mass Grashof number, Hartmann number, Prandtl number, and phase angle on the physical flow, computations are carrier out for vertical velocity, temperature and concentration and they are presented in figures.

Figure 1 represents the temperature profiles for different values of $\operatorname{Pr}(0.71,7)$. From this figure it is clear that temperature decreases with the increase of $P r$. 
In figures 2 concentration profiles are presented for different values of $S c(0.6,3.5)$ and $R(2,5)$. It is observed that increase of Schmidt number and chemical reaction parameter lead to the decrease in concentration of the species.

Velocity profiles for different values of parameters are shown in figures 3-5. Influence of $\operatorname{Gr}(2,10)$ and $\operatorname{Gm}(5,10)$ are shown in figure 3 for fixed values of $M(2), S c(0.6), R(2), \operatorname{Pr}(7)$ and $\omega t(\pi / 2)$. It is clear from the figure that velocity increases with the increase of $G r$ and $G m$. In figure 4 effects of $M(0.5,1,2), S c(0.6,3.5)$ and $R(2,5)$ are presented for some fixed values of $\operatorname{Gr}(10), \operatorname{Gm}(5), \operatorname{Pr}(7)$ and $\omega t(\pi / 2)$. It is clear from the figure that velocity decreases when $M$ and $R$ increase. Further, increase of Schmidt number $S c$ and chemical reaction parameter $R$ lead to the decrease of velocity. Velocity Profiles for different values of phase angle $\omega t$ are presented in figure 5 for some fixed values $\operatorname{Gr}(10), G m(5), \operatorname{Pr}(7), M(2), S c(0.6), R(2)$. Increase of phase angle $\omega t$ leads to decrease of velocity.

\section{Conclusions}

An exact analysis in closed form is performed to study the influence of chemically reacting hydromagnetic flow past a vertical oscillating plate. Solutions are obtained by Laplace transform technique. Some of the important conclusions of the study are as follows:

- Concentration decreases as $S c$ and $R$ increase.

- Velocity increases with increasing $G r, G m$ and decreasing $M$ and $R$.

- Also increase in $S c, R$ and $\omega t$ lead to decrease in velocity.

\section{References}

[1] Abramowitz B. M. and Stegum I. A. : Handbook of Mathematical Functional function, Dover Publications, NewYork, (1965).

[2] Chaudhary R. C. and Jain A. : MHD heat and mass diffusion flow by natural convection past a surface embedded in a porous medium, Theoret. Appl. Mech., 36(1)(2009),1-27

[3] Das U. N., Deka R. K. and Soundalgekar V. M. : Effects of mass transfer on flow past an impulsively started vertical infinite plate with constant heat flux and chemical reaction, Forschung in Ingenieurwesen, 60(1994), 284-287.

[4] Das U. N., Deka R. K. and Soundalgekar V. M. : Effect of Mass Transfer on Flow Past an Impulsively Started Infinite Vertical Plate With Chemical Reaction, The Bulletin, GUMA, 5(1998), 13-20

[5] Das U.N., Deka R.K. and Soundalgekar V.M. : Transient free convection flow past an infinite vertical plate with periodic temperature variation, Journal of Heat Transfer, Trans. ASME, 121(1999), 1091-1094.

[6] Deka R. K. and Neog B. C. : Combined effects of thermal radiation and chemical reaction on free convection flow past a vertical plate in porous medium, Adv. Appl. Fluid Mech., 6-2(2009),181-195.

[7] Deka R. K. and Neog B. C. (2009): Unsteady MHD Flow past a vertical Oscillating Plate with Thermal Radiation and Variable Mass Diffusion, Cham. J. Math, 1(2009), 79-92.

[8] Neog B. C. : Unsteady MHD Flow past a vertical Oscillating Plate with Variable Temperature and Chemical Reaction, J. As. Aca. Math., 1(2010), 97-109.

[9] Gebhart B. : Heat Transfer, Tata McGraw Hill, (1971).

[10] Gebhart B. and Pera L. : The nature of vertical natural convection flows resulting from the combined buoyancy effects of thermal and mass diffusion, Int. J. Heat and Mass Transfer, 14(1971), 2025-2050

[11] Hetnarski R. B. : An algorithm for generating some inverse Laplace transforms of exponential form, ZAMP, 26(1975), $249-253$.

[12] Muthucumaraswamy R., Ganesan P., Soundalgekar V. M. : Impulsively Started Vertical Plate with Variable Temperature and Uniform Mass Flux, The Bulletin, GUMA, 6(1999) 37-49.

[13] Muthucumaraswamy R., Meenakshisundaram S. : Theoretical Study of Chemical Reaction Effects on Vertical Oscillating Plate With Variable Temperature, Theoretical Applied Mechanics, 33(3)(2006), 245-257.

[14] Soundalgekar V. M. : Free convection effects on the Flow Past a Vertical Oscillating Plate, Astrophysics Space Science, 66(1979), $165-172$.

[15] Soundalgekar V. M. and Akolkar S. P. : Effects of free convection and mass transfer on flow past a vertical oscillating plate, Astrophysics and Space Science, 89(1983), 241-254.

[16] Soundalgekar V. M., Birajdar N. S., and Darwhekar V. K. : Mass-Transfer Effects on the Flow Past an Impulsively Started Vertical Plate with Variable Temperature or C. H. F., Astrophy. and Sp. Sc., 100(1984) 159-164

[17] Soundalgekar V. M., Lahurikar R. M., Pohanerkar S. G. and Birajdar N. S. : Effects of Mass Transfer on the Flow Past an Oscillating Infinite Vertical Plate with Constant Heat Flux, Thermophy. and AeroMech., 1(1994), 119-124.

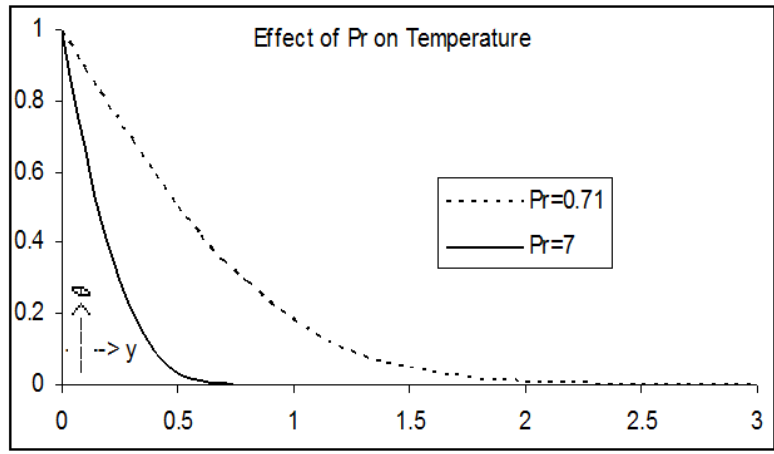

Fig-1 Temperature profiles showing the effect of $\mathrm{Pr}$.

www.iosrjournals.org 


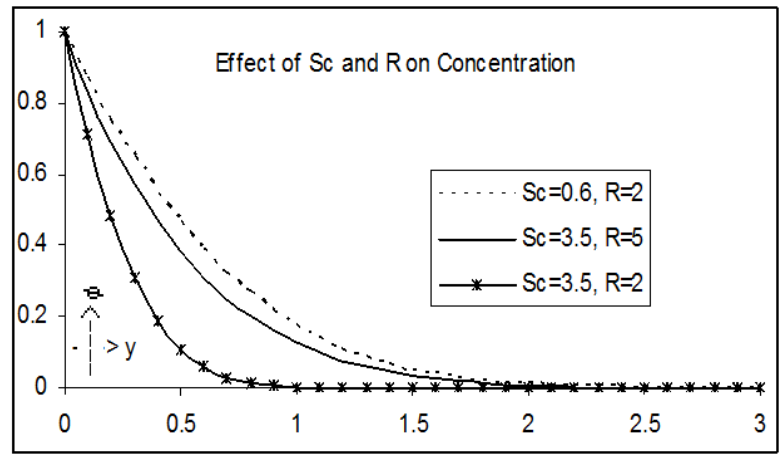

Fig-2 Concentration profiles for different values of $S c$ and $R$ at $t=0.2$.

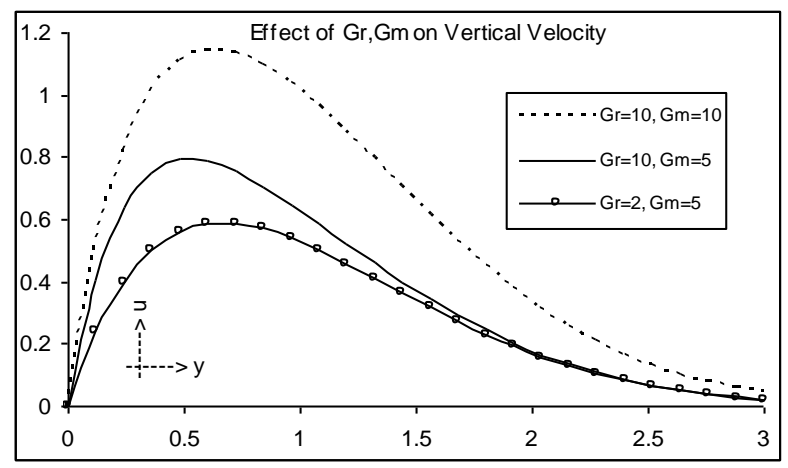

Fig-3 Velocity profiles for different values of $\mathrm{Gr}$ and $\mathrm{Gm}$.

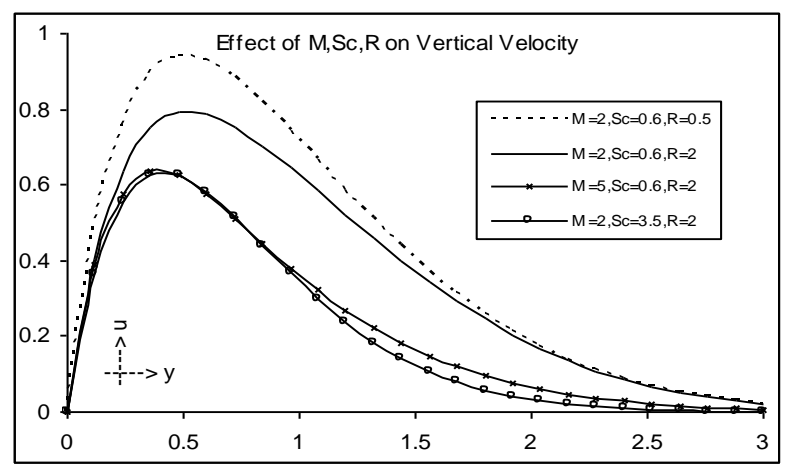

Fig-4 Velocity profiles for different values of $M, S c$ and $R$.

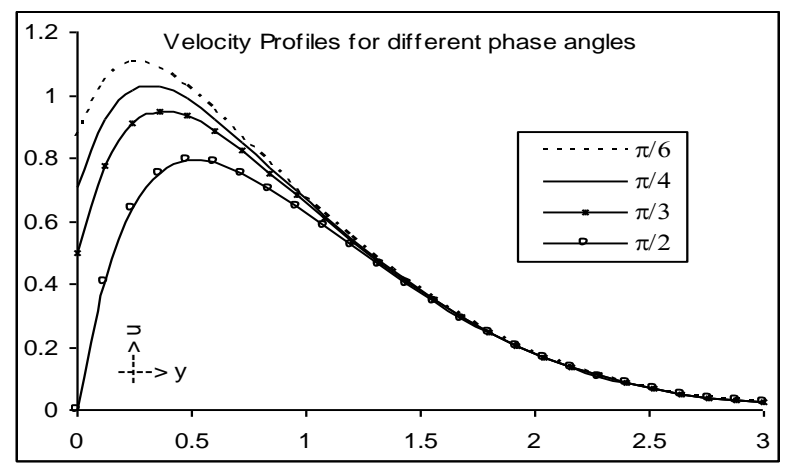

Fig- 5 Velocity profiles for different values of phase angle $\omega t$. 